\title{
High Performance 400 Gigabit Ethernet Links using Hybrid Multiband CAP/QAM Scheme
}

\author{
J. L. Wei ${ }^{1}$, Q. Cheng ${ }^{2}$, D. G. Cunningham ${ }^{3}$, R. V. Penty ${ }^{2}$, I. H. White ${ }^{2}$, H. Griesser ${ }^{1}$ \\ ${ }^{1}$ ADVA Optical Networking SE, Fraunhoferstr. 9a, 82152 Martinsried/Munich, Germany \\ Tel.: +4989890665658, Email: JWei@advaoptical.com \\ ${ }^{2}$ Centre for Photonic Systems, Electrical Engineering Division \\ ${ }^{2}$ Engineering Department, University of Cambridge, $9 \mathrm{~J}$ J Thomson Avenue, Cambridge CB3 OFA, UK \\ ${ }^{3}$ Avago Technologies, Framlingham Technology Centre, Station Road, Framlingham, Suffolk, IP13 9EZ, UK
}

\begin{abstract}
We propose the first combined $4 \times 100 \mathrm{~Gb} / \mathrm{s}$ hybrid multiband CAP-16 transmitter and QAM-16 receiver system and simulations show that it has $0.7 \mathrm{dBo}(2.2 \mathrm{dBo})$ more power margin than $8 \times 50 \mathrm{~Gb} / \mathrm{s}(4 \times 100 \mathrm{~Gb} / \mathrm{s})$ PAM-4 over DML (EML) SMF link.

OCIS codes: (060.2330) Fiber optics communications; (060.4080) Modulation.
\end{abstract}

\section{Introduction}

The exponential growth of Internet traffic continues to demand upgrading of data communication networks to higher data rates in a cost and energy efficient manner. Therefore, the IEEE P802.3bs 400GbE Task Force was created in May 2014 and agreed objectives for single-mode fiber (SMF) links including those with $500 \mathrm{~m}, 2 \mathrm{~km}$ and $10 \mathrm{~km}$ reaches. Recent research efforts have shown a number of demonstrations of various advanced modulation formats relevant alongside wavelength division multiplexing (WDM) for $400 \mathrm{GbE}$ including $112 \mathrm{~Gb} / \mathrm{s}$ four-level pulse amplitude modulation (PAM-4) over $10 \mathrm{~km} \mathrm{SMF} \mathrm{[1],} 50 \mathrm{~Gb} / \mathrm{s}$ carrierless amplitude and phase (CAP) modulation over $5 \mathrm{~km}$ SMF [2], $103 \mathrm{~Gb} / \mathrm{s}$ Duobinary transmission over $1 \mathrm{~km} \mathrm{SMF} \mathrm{[3],} 112 \mathrm{~Gb} / \mathrm{s}$ combined Duobinary-PAM4 transmission over $5 \mathrm{~km} \mathrm{SMF} \mathrm{[4],} 102.4 \mathrm{~Gb} / \mathrm{s}$ multiband CAP signal transmission over $15 \mathrm{~km} \mathrm{SMF} \mathrm{[5],} 112 \mathrm{~Gb} / \mathrm{s}$ quadrature amplitude modulation-16 (QAM-16) over $4 \mathrm{~km} \mathrm{SMF} \mathrm{link} \mathrm{[6],} \mathrm{and} 448 \mathrm{~Gb} / \mathrm{s}$ (112 Gb/s per wavelength) discrete multi-tone (DMT) over $10 \mathrm{~km} \mathrm{SMF} \mathrm{[7].} \mathrm{All} \mathrm{demonstrations} \mathrm{are} \mathrm{based} \mathrm{on} \mathrm{intensity} \mathrm{modulation} \mathrm{and} \mathrm{direct}$ detection (IMDD) for cost-effectiveness. Most of the above mentioned demonstrations, except [7], are based on externally modulated lasers (EMLs). In addition, forward error correction (FEC) is required to demonstrate the various link lengths.

Whilst the $400 \mathrm{GbE}$ Task Force is still open to the choice of modulation format and number of wavelengths per fibre, non-return-to-zero (NRZ) and PAM4 at $50 \mathrm{~Gb} / \mathrm{s}$ as well as PAM4 and DMT at $100 \mathrm{~Gb} / \mathrm{s}$ per wavelength dominate the debate. From a commercial point of view, directly modulated lasers (DMLs) are preferable to EMLs in short-haul scenarios due to their greater optical output power, compactness, low cost and low power dissipation. However, DMLs introduce nonlinearities that limit the achievable transmission performance.

In this paper, for the first time, we propose the combination of a hybrid multiband CAP transmitter [5] and a modified QAM receiver [9] scheme for 400 Gigabit Ethernet links. Both DML and EML based SMF links are analyzed. Results show that the proposed multiband hybrid CAP/QAM scheme has an excellent resistance to the dynamic nonlinearities of DMLs for bandwidths $<20 \mathrm{GHz}$ and thus allows a 4 channel solution for DML, and a strong tolerance to timing jitter due to its low symbol rate. As a result, four WDM channels each carrying $100 \mathrm{~Gb} / \mathrm{s}$ 4-band hybrid CAP-16/QAM-16 signals successfully support $10 \mathrm{~km}$ SMF transmission for both DML and EML transmitters. A reference $400 \mathrm{GbE}$ link based on PAM-4 is also investigated for comparison. Only 8 channels $\times 50$ Gb/s PAM-4 can support a DML based link. The $4 \times 100 \mathrm{~Gb} / \mathrm{s}$ hybrid 4-band CAP-16/QAM-16 system has $0.7 \mathrm{dBo}$ $(2.2 \mathrm{dBo})$ more power margin than an $8 \times 50 \mathrm{~Gb} / \mathrm{s}(4 \times 100 \mathrm{~Gb} / \mathrm{s})$ PAM-4 over DML (EML) based SMF link.

\section{System Architecture and Simulation Parameters}

The system architecture for the proposed 4-band hybrid CAP-16/QAM-16 transmitter/receiver scheme is depicted in Fig. 1. Four channels, each operating at $100 \mathrm{~Gb} / \mathrm{s}$, are considered. For each channel, the $4 \times 25 \mathrm{~Gb} / \mathrm{s}$ input bits are FEC encoded and converted to 8 parallel tributaries each being mapped to PAM-4 symbols and then pulse shaped using square-root raised cosine (SRRC) filters. The SRRC filters have 4 separate center frequencies, denoted as $f_{\text {lc }}$ to $f_{4 \mathrm{c}}$ so that four distinguishing bands are assigned. The two SRRC filters with the same center frequency form a Hilbert pair with impulse responses orthogonal to each other. The shaping filter outputs are then added and converted to an analogue signal via a digital to analogue converter (DAC) when digital implementation is considered. The resulting signal spectrum is shown in in-sets (a) and (b) of Fig. 1 (note two different roll-off coefficients of 0.5 for (a) and 1 for (b) for the SRRC filters are displayed). Power loading is adopted so that the received CAP signal has equal power on each band. After modulating a DML or EML, the optical signal is 
multiplexed with the other three channel signals that have the same transmitters. After propagating through a SMF link, the signal is de-multiplexed into 4 channels and directly detected. Each detected signal is converted into a digital signal via an analogue to digital converter (ADC). The signal is then split into 4 and each multiplies a sine and cosine sequence pair that has an oscillating frequency with the appropriate phase to match the SRRC filter center frequency. A baseband matched SRRC filter is then applied followed with a feedforward equalizer (FFE) if required. A phase rotator is applied to the equalizer output and the subsequent processing is the inverse of that in the transmitter. The system architectures for PAM-4 can be referred in [8].

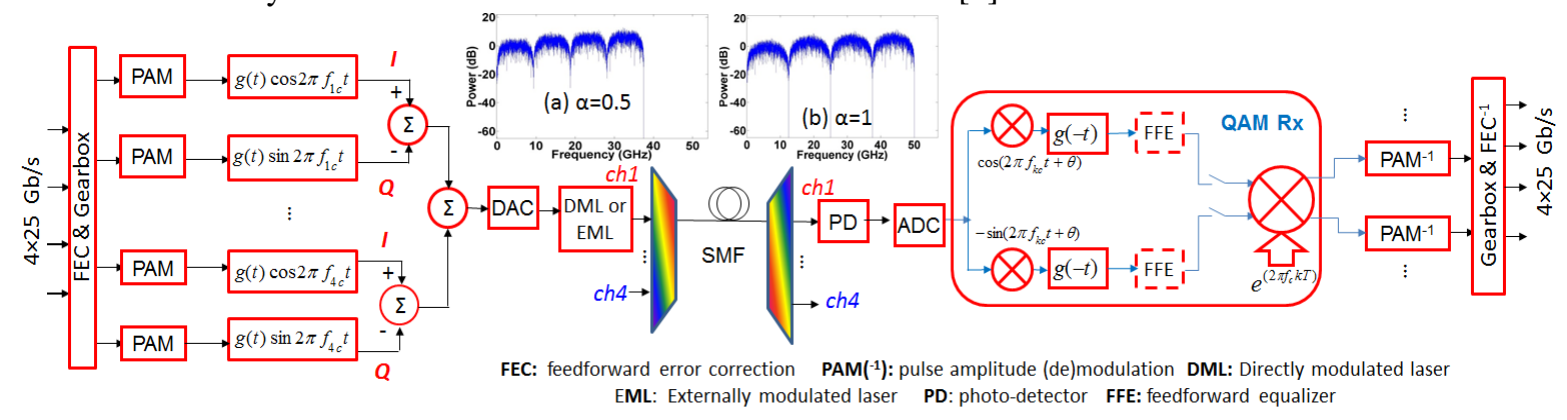

Fig. 1 System architecture diagram of a 4-band hybrid CAP/QAM transmitter/receiver scheme.

In our simulations, two types of transmitters are considered: The first is a DML modelled with simple rate equations that take into account a wide range of nonlinear effects [2] with a bias current of $50 \mathrm{~mA}$ and a peak to peak drive current amplitude of $50 \mathrm{~mA}$, which gives rise to a $3 \mathrm{~dB}$ modulation bandwidth of about $17 \mathrm{GHz}$. The second is an EML modelled as having a Gaussian response that has a rise time (20\%-80\% linear response) of $12 \mathrm{ps,}$ which corresponds to a 3-dB bandwidth of $18.6 \mathrm{GHz}$ [2]. The laser relative intensity noise (RIN) is set to -137.3 $\mathrm{dB} / \mathrm{Hz}$ for both cases. The optical receiver is modelled as a $4^{\text {th }}$ order Bessel filter with a bandwidth of $21 \mathrm{GHz}$. Using such transceivers, a reference $25 \mathrm{~Gb} / \mathrm{s}$ NRZ signal has a receiver sensitivity of $-10 \mathrm{dBm}$ at a bit error rate (BER) of $10^{-12}$ for both DML and EML cases [2]. The FEC considered here is based on the RS(544, 514) code defined in IEEE $802.3 \mathrm{bm}$, which has a FEC overhead of about $6 \%$ and a coding gain of about $7.4 \mathrm{dBe}$ for a threshold BER of $10^{-3}$. Therefore, with an optical launch power of $3 \mathrm{dBm}$, the total optical link power budget at a BER of $10^{-3}$ is 16.7 dBo. The IEEE 802.3ba wavelength specification is assumed namely four optical channels around $1310 \mathrm{~nm}$ are supported by a single fiber.

\section{Simulation results}

Figure 2 shows eye and constellation diagrams of $400 \mathrm{GbE}$ optical data links for both schemes. For $50 \mathrm{~Gb} / \mathrm{s}$ per lane PAM-4, it shows that the DML nonlinearity is negligible. In this case, two fiber pairs are required with each carrying 4 optical channel PAM-4 signals. When PAM-4 is used for $100 \mathrm{~Gb} / \mathrm{s}$ per lane, only the EML link works well while the DML link fails due to the DML nonlinearity even if equalization is used in the receiver [2]. The nonlinearity limit for a single lane $100 \mathrm{~Gb} / \mathrm{s}$ DML link can be addressed by using hybrid multiband CAP-16/QAM16 modulation. For $100 \mathrm{~Gb} / \mathrm{s}$ hybrid 4-band CAP/QAM, the DML nonlinearity is negligible. It clearly shows that a similar eye opening is obtained for both EML and DML cases either before or after equalization [Fig. 2(d) and (e)]. This is mainly because each band's CAP signal has a very low symbol rate (6.25 Gbaud without FEC).
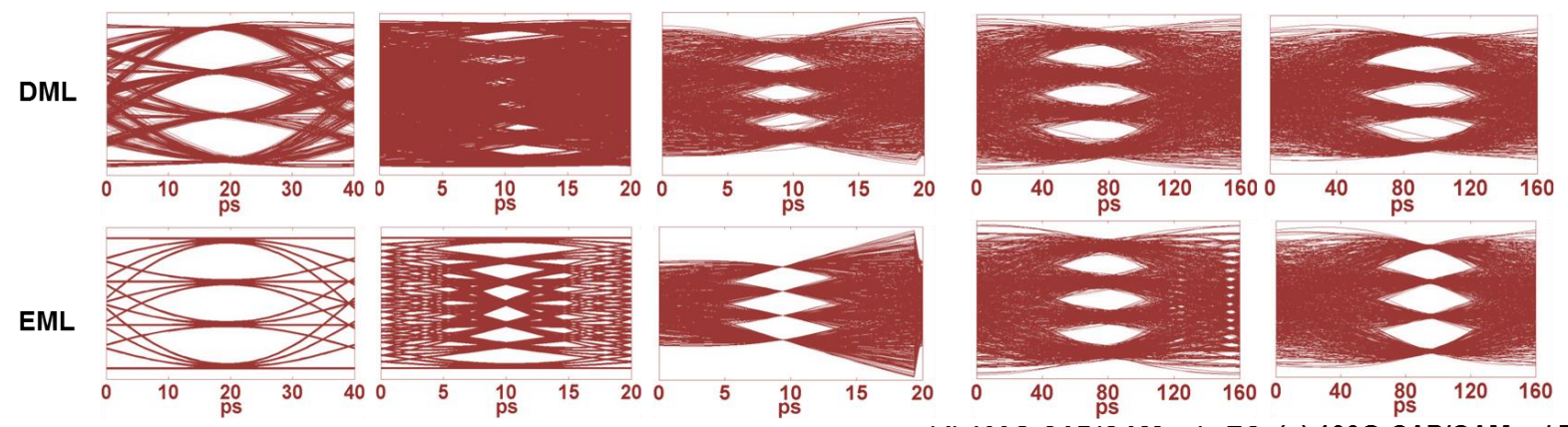

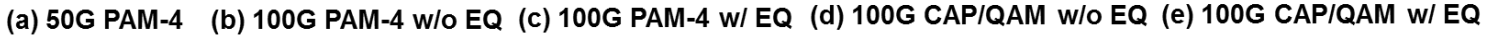

Fig. 2 Representative eye diagrams and constellation diagrams for (a) 50 Gb/s PAM-4, (b-c) 100 Gb/s PAM-4, and (d-e) the highest frequency band $100 \mathrm{~Gb} / \mathrm{s}$ 4-band hybrid CAP/QAM. The top row shows the DML case and the bottom row the EML case. The eye diagrams in (c) are based on 20 taps T/4 spaced (5 taps T spaced) trained FFE and 3 tap DFE for DML (EML) case. The eye diagrams in (e) are based on QAM receivers using a 20 taps T/4 spaced trained FFE equalizer for both DML and EML cases. 
Fig. 3 shows the system power budget of the proposed scheme using the transceivers listed in Section 2. For reference an $8 \times 50 \mathrm{~Gb} / \mathrm{s}$ PAM-4 DML link and a $4 \times 100 \mathrm{~Gb} / \mathrm{s}$ PAM-4 EML link are also investigated for comparison purposes. The link power penalty comprises contributions from the relative receiver sensitivity, dispersion penalty, RIN penalty, link loss (fiber attenuation + connector loss), deterministic timing jitter, WDM penalty and available margin (red bars). Note that the relative receiver sensitivity of hybrid CAP/QAM is offset by $3 \mathrm{dBo}$ as we assume the total per fiber launch power is identical for each scheme. The dispersion penalty for each scheme is less than $0.1 \mathrm{dBo}$ and thus is ignored. The jitter penalty is obtained by offsetting the sampling point by \pm 3 ps relative to the optimum eye opening point as shown in Fig. 1. Both DML-based systems can successfully support transmission over $10 \mathrm{~km} \mathrm{SMF}$, see Fig. 3(a). The proposed 4-band hybrid CAP/QAM exhibits the best performance with approximately $0.7 \mathrm{dBo}$ improved power margin relative to PAM-4. The excellent margin for the hybrid 4-band CAP/QAM is mainly attributed to two factors: the relative small receiver sensitivity as a result of the noise filtering by using baseband matched filter in the QAM receiver as shown in Fig. 1, and the low jitter penalty as the recovered signal has a very good horizontal and vertical eye opening and the symbol time period of the QAM receiver signal eye diagram is very large compared to the PAM-4 signal [Fig. 2]. The other penalties are similar for both schemes.

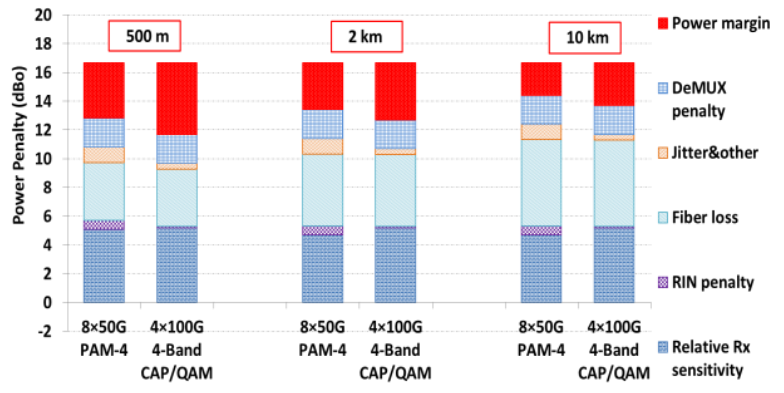

(a) DML case

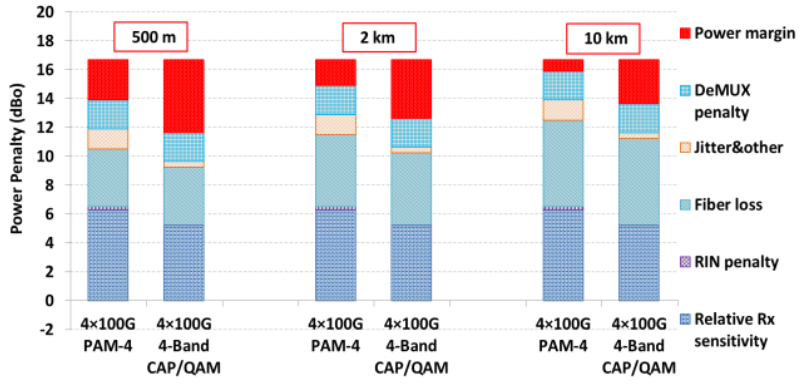

(b) EML case

Fig. 3 Link power budget for $400 \mathrm{GbE}$ links using various advanced modulation format for (a) DML case, and (b) EML case.

For comparison, as shown in Fig. 3(b), the EML-based links of the two modulation schemes are presented. PAM-4 can support $100 \mathrm{~Gb} / \mathrm{s}$ single lane data transmission over $10 \mathrm{~km}$ SMF by using a 5 tap T space FFE and 3 tap DFE, although it can only support a $50 \mathrm{~Gb} / \mathrm{s}$ single lane bit rate for DML links. EML-based hybrid 4-band CAP16/QAM-16 schemes have a power margin comparable to their DML counterparts, verifying that the DML nonlinearity is negligible due to their lower system symbol rate. This indicates that the hybrid multiband CAP/QAM is a strong contender for 400GbE. When an EML is used, the hybrid 4-band CAP-16/QAM-16 shows 2.2 dBo more margin compared with PAM-4. This is mainly attributed to the high noise enhancement from the equalization of single lane $100 \mathrm{~Gb} / \mathrm{s}$ PAM-4.

\section{Conclusion}

We have proposed and investigated $400 \mathrm{GbE}$ links enabled by $4 \times 100 \mathrm{~Gb} / \mathrm{s}$ hybrid 4-band CAP-16/QAM-16 for the first time, despite a 4-channel PAM-4 DML solution not being possible. The proposed scheme not only has excellent performance against DML nonlinearity and timing jitter but also shows $2.2 \mathrm{dBo}$ more power margin for an EMLbased system compared to a $4 \times 100 \mathrm{~Gb} / \mathrm{s}$ PAM-4 version operating over $10 \mathrm{~km} \mathrm{SMF}$.

\section{Acknowledgement}

This work was partly supported by the European Union under a Marie Curie Intra-European Fellowship for Career Development (CEEOALAN project) and by the UK EPSRC via the INTERNET project.

\section{References}

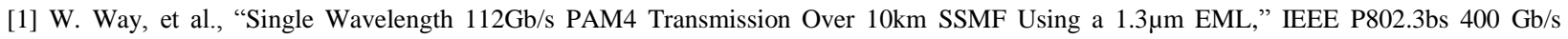
Ethernet Task Force, Sept., 2014.

[2] J. L. Wei, et al., "Performance Studies of 100 Gigabit Ethernet Enabled by Advanced Modulation Formats," IEEE Next Gen 100G Optical Ethernet Study Group, May 2012.

[3] J. Lee, et al., "Serial $103.125 \mathrm{~Gb} / \mathrm{s}$ transmission over $1 \mathrm{~km}$ SSMF for low-cost, short-reach optical interconnects," OFC'14, Paper PDP Th5A.

[4] L. F. Suhr, et al., "112-Gbit/s x 4-Lane Duobinary-4-PAM for 400GBase," ECOC, Paper Tu.4.3.2, 2014.

[5] M. I. Olmedo, et al., "Multiband Carrierless Amplitude Phase Modulation for High Capacity Optical Data Links," J. Lightw. Technol., vol. 32, no. 4, pp. 798-804, Feb. 2014..

[6] J. C. Cartledge, et al., "100 Gbit/s using intensity modulation and direct detection," ECOC, Paper We. 4. C. 3, 2013.

[7] T. Tanaka, et al., "Experimental Demonstration of 448-Gbps+ DMT Transmission over 30-km SMF," OFC, Paper M2I.5, 2014.

[8] J. L. Wei, et al., "400 Gigabit Ethernet using advanced modulation formats: performance, complexity and power dissipation," accepted by IEEE Commun. Magazine, 2014.

[9] A. H. Abdolhamid, et al., "A comparison of CAP/QAM architectures," in ISCAS '98, vol. 4, p. 316,-316/3, 1998. 